\title{
LED-based photoacoustic imaging of medical devices with carbon nanotube-polydimethylsiloxane composite coatings
}

\author{
Wenfeng Xia, ${ }^{1,2 *}$ Sacha Noimark, ${ }^{1,2,3}$ Efthymios Maneas, ${ }^{1,2}$ Mithun Kuniyil Ajith Singh, ${ }^{4}$ \\ Sebastien Ourselin, ${ }^{1,2,5}$ Simeon J. West, ${ }^{6}$ and Adrien E. Desjardins ${ }^{1,2}$ \\ ${ }^{1}$ Wellcome / EPSRC Centre for Interventional and Surgical Sciences, University College London, Charles \\ Bell House, 67-73 Riding House Street, London W1W 7EJ, United Kingdom \\ ${ }^{2}$ Department of Medical Physics and Biomedical Engineering, University College London, Gower Street, London WC1E 6BT, United Kingdom \\ ${ }^{3}$ Department of Chemistry, University College London, 20 Gordon Street, London WC1H OAJ, United Kingdom \\ ${ }^{4}$ Research and Business Development Division, PreXion Corporation, Stationsplein 45 A4.004, 3013AK Rotterdam, the Netherlands \\ ${ }^{5}$ Centre for Medical Image Computing, University College London, Gower Street, London, WC1E 6BT, United Kingdom \\ ${ }^{6}$ Department of Anaesthesia, University College Hospital, Main Theatres, Maple Bridge Link Corridor, Podium 3, 235 Euston Road, London, \\ $N W 1$ 2BU, United Kingdom \\ *wenfeng.xia@ucl.ac.uk
}

\begin{abstract}
Invasive medical devices such as needles and catheters can have low visibility with ultrasound imaging. Here, we demonstrate that visibility can be greatly improved using LEDbased photoacoustic imaging in combination with optically absorbing nanocomposite coatings.
\end{abstract}

OCIS codes: (170.5120) Photoacoustic imaging; (170.7170) Ultrasound; (160.4236) Nanomaterials.

\section{Introduction}

Ultrasound (US) imaging is widely used for guiding minimally invasive procedures. These procedures include nerve blocks, tumor biopsies and central venous catheterisations. However, invasive medical devices can have poor ultrasonic visibility, which can lead to complications such as puncture of a critical structure or injection at an incorrect location [1].

Photoacoustic (PA) imaging has been used to visualise invasive metallic medical devices including needles [2] and brachytherapy seeds $[3,4]$. A promising aspect of this paradigm is that photoacoustic signals from metallic objects can be prominent relative to those of surrounding tissues. However, excitation light sources are often bulky, which is suboptimal from a clinical translation standpoint. A second challenge is that many medical devices are nonmetallic and weakly optically absorbing, so that they may not be detectable with PA imaging.

In this study, a dual-modality PA and US system with compact light emitting diode (LED) arrays was used to image a needle and a catheter in biological tissue for the first time. To enhance PA visibility of a non-metallic epidural catheter, an elastomeric composite coating comprising multi-walled carbon nanotubes (MWCNTs) and polydimethylsiloxane (PDMS) was applied.

\section{Materials and methods}

The dual-modality LED-based PA and US imaging system (AcousticX, PreXion, Tokyo, Japan) [5] had a lineararray ultrasound imaging probe with 128 transducer elements, a central frequency of $9 \mathrm{MHz}$, and an inter-element spacing of $0.3 \mathrm{~mm}$. It alternated between two modes: a conventional B-mode ultrasound imaging mode, and a photoacoustic imaging mode in which ultrasound was generated with photoacoustic excitation light. For the latter mode, pulsed excitation light was provided by two arrays of LEDs that were affixed on opposite sides of the imaging probe. Nominally, each array provided a pulse energy of $200 \mu \mathrm{J}$ at $850 \mathrm{~nm}$ over an illumination area of 50 $\mathrm{mm} \times 7 \mathrm{~mm}$ on the sample surface; the maximum pulse repetition frequency was $4 \mathrm{kHz}$. Raw channel data from 128 channels were digitised simultaneously at $40 \mathrm{MS} / \mathrm{s}$ and images were reconstructed and displayed online at a frame rate of $6.25 \mathrm{~Hz}$, with averaging performed over 384 repeated measurements.

To evaluate the feasibility of this system for visualising medical needles and catheters, two experiments were performed. In the first, a 14 gauge metal needle (Terumo) was inserted into chicken breast tissue. In the second, an epidural catheter (Smith Medical) was dip-coated with a custom elastomeric composite material comprising functionalised MWCNTs [6] integrated in PDMS [7]. The coating was applied to the exterior of the catheter, leaving the interior uncoated and unobstructed for fluid flow. After it had cured, the catheter was positioned between layers of chicken breast tissue. In both experiments, the medical devices were positioned within the ultrasound imaging plane. 


\section{Results and discussion}

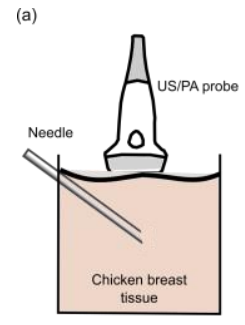

(d)

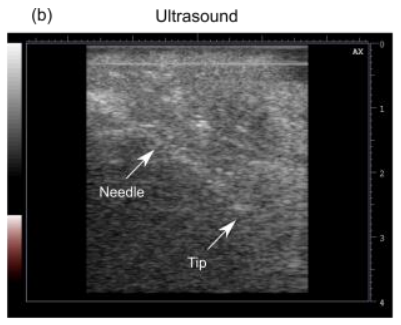

(e)

Ultrasound

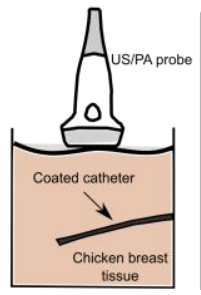

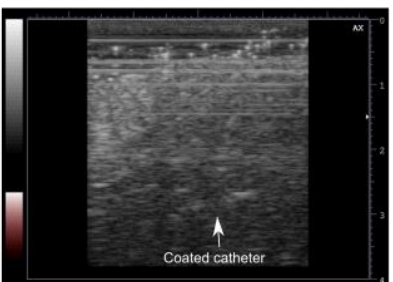

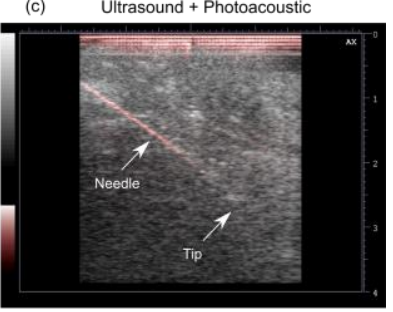

(f) Ultrasound + Photoacoustic

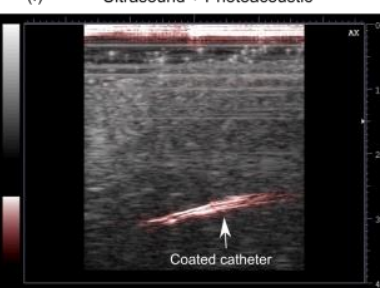

Figure 1. PA and US imaging of a needle [(a)-(c)] and a catheter with a MWCNT-PDMS composite coating [(d)-(f)] in chicken breast tissue. In overlays of the photoacoustic images on ultrasound images [(c),(f)], the medical devices are clearly visible, as compared with the ultrasound

images alone [(b),(e)]. Both PA and US images are displayed with logarithmic compression. Vertical image scales: depth in centimetres.

With conventional US imaging, the needle was barely visible [Figure 1(b)]. With PA imaging, the needle shaft was clearly visible to a depth of approximately $20 \mathrm{~mm}$ [Figure 1(c)]. Likewise, the catheter with the MWCNT-PDMS coating was not visible on the US image [Figure 1(e)], but it was clearly visible with PA imaging [Figure 1(f)]. PA signals from the coated catheter were obtained at depths greater than $30 \mathrm{~mm}$.

The maximum depth from which PA signals are obtained could be increased by delivering pulsed excitation light through the medical devices $[4,8,9]$. PA imaging provides information about the angle and shape of the medical device that is complementary to ultrasonic tracking methods, in which a miniature ultrasound sensor or transmitter within the medical device actively communicates with the external ultrasound probe [9-13].

Accurate and efficient visualisation of invasive medical devices such as catheters and needles is a long standing problem in US-guided procedures. This study demonstrated that pulsed excitation with compact LED arrays is sufficient to provide visualisation with PA imaging, and that catheters can be highly visible with PA imaging if they have an optically absorbing coating.

\section{Acknowledgements}

This work was supported by an Innovative Engineering for Health award by the Wellcome Trust (No. WT101957) and the Engineering and Physical Sciences Research Council (EPSRC) (No. NS/A000027/1), by a Starting Grant from the European Research Council (Grant No. ERC-2012-StG, Proposal 310970 MOPHIM).

\section{References}

[1] J. P. Rathmell, et al. "Safeguards to Prevent Neurologic Complications after Epidural Steroid Injections: Consensus Opinions from a Multidisciplinary Working Group and National Organizations," Surv. Anesthesiol. 60(2), 85-86 (2016).

[2] J. Su et al. "Photoacoustic imaging of clinical metal needles in tissue," J. Biomed. Opt. 15(2), 021309 (2010).

[3] M. A. L. Bell, et al. "In vivo visualization of prostate brachytherapy seeds with photoacoustic imaging," J. Biomed. Opt. 19(12), 126011 (2014).

[4] M. K. A. Singh, et al. "Photoacoustic-guided focused ultrasound for accurate visualization of brachytherapy seeds with the photoacoustic needle," J. Biomed. Opt. 21(12), 120501 (2016).

[5] A. Hariri, et al. "The characterization of an economic and portable LED-based photoacoustic imaging system to facilitate molecular imaging," Photoacoustics, in press (2017).

[6] R. J. Colchester, et al. "Laser-generated ultrasound with optical fibres using functionalised carbon nanotube composite coatings," Appl. Phys. Lett. 104, 173502 (2014).

[7] S. Noimark, et al. "Carbon-nanotube-PDMS composite coatings on optical fibers for all-optical ultrasound imaging," Adv. Funct. Mater. 26(46), 8390-8396 (2016).

[8] W. Xia, et al. "Performance characteristics of an interventional multispectral photoacoustic imaging system for guiding minimally invasive procedures," J. Biomed. Opt. 20(8), 086005 (2015).

[9] W. Xia, et al. "Ultrasonic Needle Tracking with a Fibre-Optic Ultrasound Transmitter for Guidance of Minimally Invasive Fetal Surgery," MICCAI 2017, 637-645 (2017).

[10] X. Guo, et al, "Active ultrasound pattern injection system (AUSPIS) for interventional tool guidance," PLoS One 9(10), e104262, (2014).

[11] W. Xia, et al. "In-plane ultrasonic needle tracking using a fiber-optic hydrophone," Med. Phys. 42(10), 5983-5991 (2015).

[12] W. Xia, et al. "Coded excitation ultrasonic needle tracking: An in vivo study," Med. Phys. 43(7), 4065-4073 (2016).

[13] W. Xia, et al. "Looking beyond the imaging plane: 3D needle tracking with a linear array ultrasound probe," Sci. Rep. 7, 3674-3682 (2017). 